\title{
Sergey Drobyshevich*
}

\section{ON CLASSICAL BEHAVIOR OF INTUITIONISTIC MODALITIES}

\begin{abstract}
We study connections between four types of modal operators necessity, possibility, un-necessity and impossibility-over intuitionitstic logic in terms of compositions of these modal operators with intuitionistic negation. We investigate which basic compositions, i.e. compositions of the form $\neg \delta, \delta \neg$ or $\neg \delta \neg$, yield modal operators of the same type over intuitionistic logic as over classical logic. We say that such compositions behave classically. We study which modal properties correspond to each basic compositions behaving classically over intuitionistic logic and also prove that $K C$ constitutes the smallest superintuitionistic logic over which all basic compositions behave classically.
\end{abstract}

Keywords: intuitionistic logic; modal logics; intuitionistic modalities; compositions; possibility; necessity; un-necessity; impossibility

\section{Introduction}

We study relationships between four types of modal operators, that is necessity, possibility, un-necessity and impossibility, over intuitionistic logic. The relationship of these four types of modal operators over classical logic is well known, namely, each one can be used to define any other as a composition with classical negation. For instance, the possibility operator $\diamond$-aside from being taken as principal - can be defined either by means of necessity operator $\square$ as $\neg \square \neg$, or by means of unnecessity operator $\boxminus$ as $\boxminus \neg$, or by means of impossibility operator $\diamond$ as

* This work was supported by Russian Foundation for Basic Research project RFBR-12-01-00168-a and by the Grants Council (under RF president) for State Aid of Leading Scientific Schools (grant NSh-860.2014.1). 
$\neg \vartheta$. Thus, we have twelve compositions of the form $\neg \delta, \delta \neg$ and $\neg \delta \neg$, where $\delta \in\{\square, \diamond, \boxminus, \diamond\}$, which can be regarded as natural definitions of four types of modal operators be means of each other. We call these twelve compositions basic.

Naturally, the fact that a basic composition defines an operator of some type (say, a necessity operator) consists of two conditions: firstly, this composition satisfies all properties of the corresponding modal operator and secondly, it does not satisfy any additional properties. In this work we investigate basic compositions, for which the former condition can be extended over to intuitionistic modal logics, that is which basic compositions yield the same type of modal operator over intuitionistic logic as over classical logic. We say that such compositions behave classically over intuitionistic logic. It turns out that five of twelve basic compositions behave classically over intuitionistic logic and, since non of them define basic modal operators over intuitionistic logic, for these five compositions we obtain axiomatizations in terms of modal operators of the corresponding type. We also find the smallest superintuitionistic logic, over which every basic composition behaves classically.

The natural interest to this problem stems from the fact that over intuitionistic logic four types of modal operators are not dual to each other. For classical logic these dualities essentially mean that we can reduce the study of basic properties of four types of modal operators to study of a single logic, that is the smallest classical normal modal logic $K$, and its different definitional variants. On the contrary there does not exist a single general framework for investigating intuitionistic modalities and a number of different approaches were introduced. We mention most general of them. One such approach is due to V. Sotirov [17], who introduced an intuitionistic modal logic IML with a single abstract modality $M$, which satisfies only the extensionality (or congruence) principle

$$
\frac{\varphi \leftrightarrow \psi}{M \varphi \leftrightarrow M \psi} .
$$

This way four types of modal operators can be obtained by extending $I M L$ with some new axioms. For some development of this idea, including algebraic semantics for such systems, see for example [19]. Another approach, and the one we will use in this paper, is due to M. Božić and K. Došen, who introduced four different systems $H K \square, H K \diamond, H K \boxminus$ and $H K \diamond$ corresponding to each type of modal operators, which, for brevity, we will collectively call Heyting-Kripke logics. Logics HK $\square$ and 
$H K \diamond$ were introduced in [2] and logics $H K \diamond$ and $H K \boxminus$ in [4]. See also [5] for study of some extensions of Heyting-Kripke logics.

We mention some related results. F. Servi (see [9], [10] and [11]) developed an intuitionistic modal logic FS in the language containing both $\square$ and $\diamond$, which has some weak connection between these modalities and can be regarded as an intuitionistic analogue of $K$ in terms of its relation to Gödel translation and to the standard translation into first order language. In [15] it was shown that there exists exactly 31 nonequivalent modalities, defined as compositions of necessity operator and intuitionistic negation, in logic IM4 which is an intuitionistic analogue of $S 4$. In [12] properties of composition $\neg \square \neg$ in some extensions of IM4 were studied as well as non-equivalent modalities in these systems. A number of results on negative modalities stem from the theory of negation. For instance, impossibility and un-necessity operators over intuitionistic logic were studied by D. Vakarelov in [18]. More specifically, impossibility and un-necessity operators correspond to normal and conormal negations studied by Vakarelov over distributive logics. In [3] an intuionistic modal logic $N^{*}$ was introduced the only modal operator $\sim$ in which is the smallest operator over intuitionistic logic which is both unnecessity and impossibility operator. In [6] composition $\sim \sim$ was studied in $N^{*}$ and its axiomatization as a necessity operator was obtained. In [7] composition $\neg \sim$, which is also a necessity operator was studied in $N^{*}$. As a part of this problem an axiomatization of composition $\neg \boxminus$ in logic $H K \boxminus$ as a necessity operator was obtained, which is one of the above mentioned basic compositions. It is exactly this result that motivated the current study. For a general overview of intuitionistic modal logics see for example [20].

The work consists of two parts. In the first part some preliminary notions and results are given. In Section 1.1 Heyting-Kripke logics are defined along with some necessary notions. In Section 1.2 Kripke semantics for Heyting-Kripke logics are given and the canonical method of proving completeness wrt to corresponding frames is outlined. In Section 1.3 algebraic semantics are presented for both Heyting-Kripke logics and its normal extensions. Finally, in Section 1.4 the problem we will be solving is formulated along with some necessary notions. It is also shown which basic compositions behave classically over intuitionistic logics and some additional preliminary results are proven.

The second part contains all main results. Sections 2.1-2.4 dedicated to axiomatization of those basic compositions, which behave classically 
over intuitionistic logic, excluding composition $\neg \boxminus$. Composition $\neg \boxminus$ was previously considered in [7] and its axiomatization as a necessity operator was obtained in form of logic

$$
H K(\neg \boxminus)=H K \square+\neg \neg \square p \rightarrow \square p .
$$

We adopt the technique developed in [7] to obtain axiomatizations of all other basic compositions behaving classically over intuitionistic logic. The method is based on embedding of logics via natural translations and on reduction of Kripke semantics of logics. As it turns out all axiomatization are obtained by adding very similar formulas.

Finally in Section 4.5 we turn to find the smallest superintuitionistic logic over which all basic compositions behave classically. It turns out that this logic is $K C$ which can be obtained by adding to intuitionistic logic the weak law of excluded middle $\neg p \vee \neg \neg p$. There is quite a number of things to like about $K C$. It is both the largest superintuitionistic logic, which shares its positive fragment with intuitionistic logic, and the smallest superintuitionistic logic in which each formula has negation-free equivalent (wrt to inference relation) formula [21]. Moreover, it is the only superintuitionistic logic which has both these properties. $K C$ has both finite model property and Craig's interpolation property (as well as stronger Lyndon interpolation property), see [14] and [13]. The weak law of excluded middle is also conservative over intuitionistic logic, meaning we can substitute only propositional variables in it when inferring a formula [13]. From this it follows that addition of the weak law of excluded middle preserves Craig's interpolation property and finite model property over intuitionistic logic.

\section{Preliminaries}

\subsection{Definitions of logics}

Consider a language $\mathcal{L}=\{\wedge, \vee, \rightarrow, \neg\}$ consisting of logical connectives of conjunction, disjunction, implication and negation, respectively. By $\mathcal{L} \delta$ denote $\mathcal{L}$ augmented with the symbol $\delta \in\{\square, \diamond, \boxminus, \diamond\}$, where $\square$ stands for modal necessity operator, $\diamond$ - for possibility, $\boxminus$ - for un-necessity and $\vartheta$ - for impossibility. The set of all formulas For $\mathcal{L} \delta$ of the language $\mathcal{L} \delta$ is formed the standard way from the fixed countable set of propositional variables Prop using logical connectives and modal operator $\delta$. In case 
it will be clear from the context, we will not specify the langauge of formulas in consideration.

For the rest of the work symbol $\delta$ will denote one of the modal operators $\square, \diamond, \boxminus$ or $\vartheta$. We will specify which one of them is currently being considered only when it will be necessary.

The rule of the form

$$
\frac{\varphi \rightarrow \psi}{\nabla \varphi \rightarrow \nabla \psi}
$$

we will call the monotonic rule for $\nabla$ and the rule

$$
\frac{\varphi \rightarrow \psi}{\nabla \psi \rightarrow \nabla \varphi}
$$

we will call the contraposition rule for $\nabla$, where $\nabla$ is an unary connective.

We will identify a logic with its set of theorems. Thus by a logic in language $\mathcal{L} \delta$ we will call a set of formulas of said language, which is closed under both modus ponens and the substitution rule. A normal logic is a logic, which is additionally closed under the monotonic rule for $\delta$ in case $\delta \in\{\square, \diamond\}$ and under the contraposition rule for $\delta$ in case $\delta \in\{\ominus, \boxminus\}$. The class of all extensions of a given logic $L$ (i.e. of logics which contain $L$ as a subset) we will denote Ext $L$ and the class of its normal extensions (i.e. of normal logics containing $L$ ) we will denote NExt $L$. As usual non-trivial extensions of intuitionistic logic we will call superintuitionistic logics.

Let us define Heyting-Kripke logics. By $H K \delta$ we will denote the smallest normal logic in the language $\mathcal{L} \delta$ containing all theorems of intuitionistic logic together with formulas $\delta 1$ and $\delta 2$, where:

$\square$ 1. $\square(p \rightarrow p)$;

$\square 2 . \square p \wedge \square q \rightarrow \square(p \wedge q)$;

$\diamond 1 . \ominus \neg(p \rightarrow p)$;

$\diamond 2 . \quad p \wedge \diamond q \rightarrow \diamond(p \vee q)$ $\diamond 1 . \neg \diamond \neg(p \rightarrow p) ;$

$\diamond 2 . \diamond(p \vee q) \rightarrow \diamond p \vee \diamond q$

$\boxminus 1 . \neg \boxminus(p \rightarrow p)$;

$\boxminus 2 . \boxminus(p \wedge q) \rightarrow \boxminus p \vee \boxminus q$

By $L+\varphi$ for a normal logic $L$ we will denote the smallest normal logic containing both $L$ and $\varphi$. We will use similar denotation for superintuitionistic logic $L$ and $\delta$-free formula $\varphi$.

Let us introduce an abbreviation $\varphi \leftrightarrow \psi:=(\varphi \rightarrow \psi) \wedge(\psi \rightarrow \varphi)$. It is easy to show using axioms of intuitionistic logic and the correspond- 
ing modal rule that all normal extensions of $H K \delta$ are closed under the replacement rule

$$
\frac{\varphi \leftrightarrow \psi}{\chi(\varphi) \leftrightarrow \chi(\psi)}
$$

Let us also define the usual way the intuitionistic falsity constant $\perp:=\neg\left(p_{0} \rightarrow p_{0}\right)$. Thus, in any $L \in$ NExt $H K \delta$ we have $\neg \varphi \leftrightarrow(\varphi \rightarrow$ $\perp) \in L$.

With every $\operatorname{logic} L$ we will associate the inference relation $\vdash_{L}$. More specifically, for a set of formulas $\Gamma \cup\{\varphi\}$ we will write $\Gamma \vdash_{L} \varphi$ iff $\varphi$ can be inferred from formulas in $L \cup \Gamma$ using modus ponens. We will write $\Gamma, \varphi_{1}, \ldots, \varphi_{n} \vdash_{L} \psi$ instead of $\Gamma \cup\left\{\varphi_{1}, \ldots, \varphi_{n}\right\} \vdash_{L} \psi$ and $\vdash_{L} \psi$ instead of $\varnothing \vdash_{L} \psi$. For sets of formulas $\Gamma$ and $\Delta$ by $\Gamma \vdash_{L} \Delta$ we denote the fact that $\Gamma \vdash_{L} \psi_{1} \vee \cdots \vee \psi_{n}$ for some formulas $\psi_{1}, \ldots, \psi_{n} \in \Delta$. We will omit prefix $L$ in case the logic in consideration will be clear from the context.

Notice that for $L \in$ NExt $H K \delta$ classical deduction theorem holds wrt $\vdash_{L}$. Moreover, we have

$$
\Gamma, \varphi_{1}, \ldots, \varphi_{n} \vdash_{L} \psi \Longleftrightarrow \Gamma \vdash_{L}\left(\varphi_{1} \wedge \cdots \wedge \varphi_{n}\right) \rightarrow \psi
$$

for a set of formulas $\Gamma$ and formulas $\varphi_{1}, \ldots, \varphi_{n}, \psi$.

\subsection{Kripke semantics for Heyting-Kripke logics}

We proceed by introducing Kripke-style semantics for Heyting-Kripke logics. Proofs of all results of the section concerning positive modalities can be found in [2] and those concerning negative modalities in [4].

Definition 1. A tuple $\mathcal{W}=\langle W, \leq, R\rangle$ is an $H K \delta$-frame if $\langle W, \leq\rangle$ is a non-empty partially ordered set and $R \subseteq W^{2}$ is an accessibility relation satisfying the following interplay condition:

$$
\begin{aligned}
& \forall x, y, z \in W(x \leq y \text { and } y R z \Rightarrow x R z) \text { in case } \delta \in\{\square, \nabla\} \\
& \forall x, y, z \in W(x \leq y \text { and } x R z \Rightarrow y R z) \text { in case } \delta \in\{\oslash, \boxminus\}
\end{aligned}
$$

A pair $\mu=\langle\mathcal{W}, \mathbf{v}\rangle$ is an $H K \delta$-model if $\mathcal{W}$ is an $H K \delta$-frame and $\mathbf{v}:$ Prop $\rightarrow 2^{W}$ is a valuation satisfying the heredity condition

$$
\forall x, y \in W(x \in \mathbf{v}(p) \text { and } x \leq y \Rightarrow y \in \mathbf{v}(p)) .
$$

In this case we say that $\mu$ is a model over $H K \delta$-frame $\mathcal{W}$. 
A valuation can be extended to obtain the validity relation on the worlds of a model:

$$
\begin{aligned}
& \mu, x \vDash p \quad \Longleftrightarrow x \in \mathbf{v}(p) \text { for } p \in \text { Prop; } \\
& \mu, x \vDash \varphi \wedge \psi \quad \Longleftrightarrow \mu, x \vDash \varphi \text { and } \mu, x \vDash \psi \text {; } \\
& \mu, x \vDash \varphi \vee \psi \Longleftrightarrow \mu, x \vDash \varphi \text { or } \mu, x \vDash \psi ; \\
& \mu, x \vDash \neg \varphi \quad \Longleftrightarrow \forall y \geq x \mu, y \not \models \varphi ; \\
& \mu, x \vDash \varphi \rightarrow \psi \Longleftrightarrow \forall y \geq x(\mu, y \vDash \varphi \Rightarrow \mu, y \vDash \psi) ; \\
& \mu, x \vDash \square \varphi \quad \Longleftrightarrow \quad \forall y \in W(x R y \Rightarrow \mu, y \vDash \varphi) \text {; } \\
& \mu, x \vDash \Delta \varphi \quad \Longleftrightarrow \quad \exists y \in W(x R y \text { and } \mu, y \vDash \varphi) \text {; } \\
& \mu, x \vDash \nabla \varphi \quad \Longleftrightarrow \forall y \in W(x R y \Rightarrow \mu, y \not \models \varphi) \text {; } \\
& \mu, x \vDash \boxminus \varphi \quad \Longleftrightarrow \quad \exists y \in W(x R y \text { and } \mu, y \not \models \varphi) \text {. }
\end{aligned}
$$

Suppose $\varphi \in$ For $\mathcal{L} \delta$. For an $H K \delta$-model $\mu=\langle W, \leq, R, \mathbf{v}\rangle$ we denote by $\mu \vDash \varphi$ the fact that $\mu, x \vDash \varphi$ holds for all $x \in W$. Similarly for an $H K \delta$-frame $\mathcal{W}$ the fact that $\mu \vDash \varphi$ holds for any $H K \delta$-model $\mu$ over $\mathcal{W}$ is denoted by $\mathcal{W} \vDash \varphi$. Finally for a set of formulas $\Gamma$ we write $\mu \vDash \Gamma$ $(\mathcal{W} \vDash \Gamma)$ if $\mu \vDash \varphi(\mathcal{W} \vDash \varphi)$ holds for every $\varphi \in \Gamma$.

Let $L \in$ NExt $H K \delta$. An $H K \delta$-frame $\mathcal{W}$ is an $L$-frame, if $\mathcal{W} \vDash L$. $H K \delta$-models over $L$-frames we will call $L$-models.

As usual the heredity condition can be generalized the following way

Proposition 1. For an arbitrary $H K \delta$-model $\mu=\langle W, \leq, R, \mathbf{v}\rangle$ and formula $\varphi$ we have

$$
\forall x, y \in W(\mu, x \vDash \varphi \text { and } x \leq y \Rightarrow \mu, y \vDash \varphi) .
$$

Completeness of Heyting-Kripke logics wrt corresponding types of frames can be proved using the canonical models method. Let us outline it for future use.

Let $L \in$ NExt $H K \delta$. A set of formulas $\Gamma$ is a prime $L$-theory if i) $L \subseteq$ $\Gamma$; ii) $\Gamma$ is closed under modus ponens; iii) $\Gamma$ is non-trivial, i.e. $\Gamma \neq$ For $\mathcal{L} \delta$; iv) $\Gamma$ satisfies disjunction property, i.e. $\varphi \vee \psi \in \Gamma$ implies $\varphi \in \Gamma$ or $\psi \in \Gamma$ for any formulas $\varphi$ and $\psi$. We then have

Lemma 1 (extension lemma). For any $L \in$ NExt $H K \delta$ and sets of formulas $\Gamma$ and $\Delta \neq \varnothing$ if $\Gamma \nvdash_{L} \Delta$, then a prime $L$-theory $\Gamma^{\prime}$ exists such that $\Gamma \subseteq \Gamma^{\prime}$ and $\Gamma^{\prime} \nvdash_{L} \Delta$. 
Corollary 1. Let $L \in$ NExt $H K \delta$. Any $L$-consistent set of formulas $\Gamma$ (i.e. such that $\Gamma \nvdash_{L} \perp$ ) is contained in some prime $L$-theory.

Definition 2. Let $L \in$ NExt $H K \delta$. The canonical $L$-frame is a triple $\mathcal{W}^{c}=\left\langle W^{c}, \leq^{c}, R^{c}\right\rangle$, where i) $W^{c}$ is the set of all prime $L$-theories; ii) $\leq^{c}$ is the set-theoretic inclusion relation $\subseteq$ on $W^{c}$; iii) $\Gamma R^{c} \Delta$ holds for $\Gamma, \Delta \in$ $W^{c}$ iff

$$
\begin{aligned}
& \forall \varphi(\square \varphi \in \Gamma \Rightarrow \varphi \in \Delta) \text { in case } \delta=\square ; \\
& \forall \varphi(\diamond \varphi \notin \Gamma \Rightarrow \varphi \notin \Delta) \text { in case } \delta=\diamond ; \\
& \forall \varphi(\nabla \varphi \in \Gamma \Rightarrow \varphi \notin \Delta) \text { in case } \delta=\diamond ; \\
& \forall \varphi(\boxminus \varphi \notin \Gamma \Rightarrow \varphi \in \Delta) \text { in case } \delta=\boxminus
\end{aligned}
$$

The canonical L-model is a pair $\mu^{c}=\left\langle\mathcal{W}^{c}, \mathbf{v}^{c}\right\rangle$ consisting of the canonical $L$-frame together with a valuation $\mathbf{v}^{c}$ such that

$$
\forall \Gamma \in W^{c}\left(\Gamma \in \mathbf{v}^{c}(p) \Longleftrightarrow p \in \Gamma\right) .
$$

Notice that the definition above is sound in the sense that canonical models are indeed models of the corresponding type.

Lemma 2 (canonical models). Let $L \in$ NExt $H K \delta$ and $\mu^{c}=\left\langle W^{c}, \leq^{c}\right.$, $\left.R^{c}, \mathbf{v}^{c}\right\rangle$ be the canonical $L$-model, then for any $\Gamma \in W^{c}$ and formula $\varphi$ the following equivalence holds:

$$
\mu^{c}, \Gamma \vDash \varphi \Longleftrightarrow \varphi \in \Gamma \text {. }
$$

We say that a $\operatorname{logic} L \in$ NExt $H K \delta$ is complete wrt a class $\mathcal{K}$ of $H K \delta$-frames if

$$
L=\{\varphi \mid \mathcal{W} \vDash \varphi \text { for all } \mathcal{W} \in \mathcal{K}\} .
$$

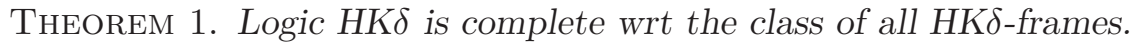

Let us remind that a logic $L \in$ NExt $H K \delta$ is canonical if the canonical $L$-frame is indeed an $L$-frame. Any canonical logic is complete with respect to some class of $H K \delta$-frames (for instance with respect to the class containing only the canonical $L$-frame).

\subsection{Algebraic semantics for Heyting-Kripke logics}

The following section concerns algebraic semantics for Heyting-Kripke logics. Algebraic semantics for logics with positive modal operators were 
developed in [19], while semantics for negative ones can be traced back to the works of D. Vakarelov [18] and were more recently formulated in [8]. While [19] does not contain the results of the section as formulated here, all of them can be proved by analogy with the results in [8].

Definition 3. Let $\mathfrak{A}=\langle A, \vee, \wedge, \rightarrow, \neg, 0,1\rangle$ be a Heyting algebra, then we say that

1. an algebra $\mathfrak{A} \square=\langle A, \vee, \wedge, \rightarrow, \neg, \square, 0,1\rangle$ is a $\square$-algebra if $\square$ is an unary operation on $A$ and identities $\square(x \wedge y)=\square x \wedge \square y$ and $\square 1=1$ hold on $\mathfrak{A}$;

2. an algebra $\mathfrak{A}^{\diamond}=\langle A, \vee, \wedge, \rightarrow, \neg, \diamond, 0,1\rangle$ is a $\diamond$-algebra if $\diamond$ is an unary operation on $A$ and identities $\diamond(x \vee y)=\diamond x \vee \diamond y$ and $\diamond 0=0$ hold on $\mathfrak{A}$;

3. an algebra $\mathfrak{A}^{\boxminus}=\langle A, \vee, \wedge, \rightarrow, \neg, \boxminus, 0,1\rangle$ is a $\boxminus$-algebra if $\boxminus$ is an unary operation on $A$ and identities $\boxminus(x \wedge y)=\boxminus x \vee \boxminus y$ and $\boxminus 1=0$ hold on $\mathfrak{A}$;

4. an algebra $\mathfrak{A}^{\ominus}=\langle A, \vee, \wedge, \rightarrow, \neg, \diamond, 0,1\rangle$ is a $\diamond$-algebra if $\diamond$ is an unary operation on $A$ and identities $\diamond(x \vee y)=\diamond x \wedge \diamond y$ and $\diamond 0=1$ hold on $\mathfrak{A}$.

By $\leq$ we will denote the lattice ordering on $\delta$-algebra $\mathfrak{A}$. Notice that this denotation does not specify algebra in consideration as it will usually be clear from the context. An expression $(a \rightarrow b) \wedge(b \rightarrow a)$ we will abbreviate as $a \leftrightarrow b$. As for Heyting algebras $a \rightarrow b=1$ is equivalent to $a \leq b$ and $a \leftrightarrow b=1$ is equivalent to $a=b$ for all $a, b \in A$. Moreover, $a \leq b$ implies $\delta a \leq \delta b$ in case $\delta \in\{\square, \diamond\}$ and $\delta b \leq \delta a$ in case $\delta \in\{\boxminus, \nabla\}$.

As usual, the class $\mathcal{V}^{\delta}$ of all $\delta$-algebras forms a variety. By $\mathrm{Eq}(\mathcal{V})$ we denote the equational theory of a variety $\mathcal{V}$, i.e. the set of all identities which hold on all algebras in $\mathcal{V}$.

Let $\mathfrak{A}$ be a $\delta$-algebra. An $\mathfrak{A}$-valuation is a homomorphism from algebra of formulas of the language $\mathcal{L} \delta$ to $\mathfrak{A}$. We write $\mathfrak{A} \vDash \varphi$ if $v(\varphi)=1$ for every $\mathfrak{A}$-valuation $v$, where 1 is the unit element of $\mathfrak{A}$. It is clear that $\mathfrak{A} \vDash \varphi$ iff $\varphi=1$ is an identity on $\mathfrak{A}$ (with propositional variables being considered as variables over elements of $\mathfrak{A})$.

For any $\delta$-algebra $\mathfrak{A}$ and any class of $\delta$-algebras $\mathcal{K}$ put

$$
L \mathfrak{A}:=\{\varphi \mid \mathfrak{A} \vDash \varphi\}, \quad L \mathcal{K}:=\bigcap\{L \mathfrak{A} \mid \mathfrak{A} \in \mathcal{K}\} .
$$

It is easy to show that $L \mathfrak{A}$ and $L \mathcal{K}$ form normal extensions of $H K \delta$. 
The lattice of sub-varieties of a variety $\mathcal{V}$ we will denote $\operatorname{Sub}(\mathcal{V})$. Then for $L \in \operatorname{NExt} H K \delta$ and $\mathcal{V} \in \operatorname{Sub}\left(\mathcal{V}^{\delta}\right)$ put

$$
V(L):=\{\mathfrak{A} \mid L \subseteq L \mathfrak{A}\} \quad \text { and } \quad L(\mathcal{V}):=\{\varphi \mid \varphi=1 \in \operatorname{Eq}(\mathcal{V})\} .
$$

Naturally we have $V(L) \in \operatorname{Sub}\left(\mathcal{V}^{\delta}\right)$ and $L(\mathcal{V}) \in$ NExt $H K \delta$. Moreover,

TheOrem 2. The mappings $V: \operatorname{NExt} H K \delta \rightarrow \operatorname{Sub}\left(\mathcal{V}^{\delta}\right)$ and $L: \operatorname{Sub}\left(\mathcal{V}^{\delta}\right)$ $\rightarrow$ NExt $H K \delta$ form mutually inverse dual lattice isomorphisms between NExt $H K \delta$ and $\operatorname{Sub}\left(\mathcal{V}^{\delta}\right)$. In particular,

1. for any $L \in \operatorname{NExt} H K \delta$ we have $\varphi \in L$ iff $\varphi=1 \in \operatorname{Eq}(V(L))$;

2. for any $\mathcal{V} \in \operatorname{Sub}\left(\mathcal{V}^{\delta}\right)$ we have $\mathcal{V}=V(L(\mathcal{V}))$.

\subsection{The problem}

By a $\delta$-composition we will call any sequence $\nabla=\alpha_{1} \ldots \alpha_{n}$ with $\alpha_{i} \in$ $\{\delta, \neg\}$. We will sometimes call a $\delta$-compositions simply compositions when it will not be of importance which modal operator is denoted by $\delta$.

It is well known that each of the four types of modal operators over classical logic can be defined using any other with the help of classical negation. In particular, over classical logic we have

1. compositions $\neg \diamond \neg, \neg \boxminus$ and $\diamond \neg$ form necessity operators;

2. compositions $\neg \square \neg, \neg \vartheta$ and $\boxminus \neg$ form possibility operators;

3. compositions $\neg \diamond \neg, \neg \square$ and $\diamond \neg$ form un-necessity operators;

4. compositions $\neg \boxminus \neg, \neg \diamond$ and $\square \neg$ form impossibility operators.

Twelve compositions listed above we will call basic compositions.

Let $\rho \in\{\square, \diamond, \boxminus, \nabla\}$ and $\nabla$ be a $\rho$-composition. Let us define a translation $(\cdot)^{\nabla}$ from For $\mathcal{L} \delta$ to For $\mathcal{L} \rho$, which essentially replaces $\delta$ with $\nabla$ in formulas of the language $\mathcal{L} \delta$ :

$$
\begin{aligned}
& (p)^{\nabla}:=p \text { for } p \in \text { Prop; } \\
& (\varphi \circ \psi)^{\nabla}:=(\varphi)^{\nabla} \circ(\psi)^{\nabla} \text { for } \circ \in\{\wedge, \vee, \rightarrow\} ; \\
& (\neg \varphi)^{\nabla}:=\neg(\varphi)^{\nabla} ; \\
& (\delta \varphi)^{\nabla}:=\nabla(\varphi)^{\nabla} .
\end{aligned}
$$

For a set of formulas $\Gamma$ put $(\Gamma)^{\nabla}:=\left\{(\varphi)^{\nabla} \mid \varphi \in \Gamma\right\}$.

Let $L \in$ NExt $H K \delta$ and $\rho \in\{\square, \diamond, \boxminus, \diamond\}$. We say that a $\delta$-composition $\nabla$ is a $\rho$-operator (operator of type $\rho$ ) in logic $L$ if the set $(H K \rho)^{\nabla}$ is a 
subset of the $\langle\wedge, \vee, \rightarrow, \neg, \nabla\rangle$-fragment of logic $L$. It is easy to see that this is equivalent to the fact that formulas $(\rho 1)^{\nabla}$ and $(\rho 2)^{\nabla}$ are theorems of $L$ and $L$ is closed under the corresponding modal rule for $\nabla$ (under the monotonic rule in case $\rho \in\{\square, \diamond\}$ and the contraposition rule in case $\rho \in\{\boxminus, \diamond\})$.

We will say that a composition $\nabla$ behaves classically in $L$ if $\nabla$ is an operator of the same type in $L$ as over classical logic. For instance $\neg \diamond \neg$ behaves classically in $L$ if it is a $\square$-operator in $L$ and $\neg \diamond$ behaves classically in $L$ if it is a $\diamond$-operator in $L$ (see the list in the beginning of the section).

Let $L$ be a superintuitionistic logic. Denote by $L \delta$ the smallest normal extension of $H K \delta$ containing $L$ (thus $\operatorname{Int} \delta=H K \delta$ ). We will say that a $\delta$-composition $\nabla$ behaves classically over $L$ if it behaves classically in $L \delta$. Thus a $\delta$-composition $\nabla$ behaves classically over Int iff it behaves classically in $H K \delta$.

Proposition 2. 1. Compositions $\square \neg, \neg \diamond, \neg \boxminus, \boxminus \neg$ and $\neg \boxminus \neg$ behave classically over Int.

2. Compositions $\neg \square, \diamond \neg, \neg \vartheta, \diamond \neg, \neg \square \neg, \neg \diamond \neg$ and $\neg \diamond \neg$ do not behave classically over Int.

Proof. 1. Can be proved routinely using completeness theorems of Heyting-Kripke logics wrt corresponding Kripke semantics.

2. Suppose $\nabla=\neg \diamond \neg$, we then need to show that $\nabla$ is not a $\square$ operator in $H K \diamond$. Let us consider an $H K \diamond$-frame $\mathcal{W}_{1}=\left\langle W_{1}, \leq_{1}, R_{1}\right\rangle$ depicted as

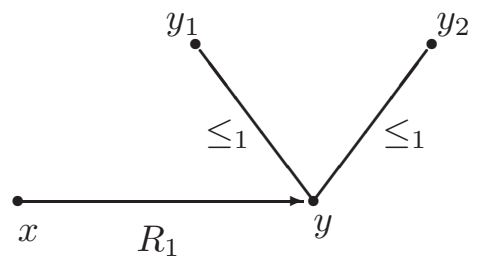

and put $\mu_{1}=\left\langle\mathcal{W}_{1}, \mathbf{v}_{1}\right\rangle$, where $\mathbf{v}_{1}(p)=\left\{y_{1}\right\}$ and $\mathbf{v}_{1}(q)=\left\{y_{2}\right\}$. Then it is easy to check that $\mu_{1}, x \not \models \nabla p \wedge \nabla q \rightarrow \nabla(p \wedge q)=(\square 2)^{\nabla}$ and thus $(\square 2)^{\nabla} \notin H K \diamond$, which means that $\nabla$ does not behave classically in $H K \diamond$. Notice also that nevertheless $(\square 1)^{\nabla}$ is a theorem of $H K \diamond$ and that $H K \diamond$ is closed under the monotonic rule for $\nabla$. The case of composition $\vartheta \neg$ is considered similarly using the same frame. 
Suppose now $\nabla=\neg \square$. We will show that $\nabla$ is not an $\boxminus$-operator in $H K \square$. Consider an $H K \square$-frame $\mathcal{W}_{2}=\left\langle W_{2}, \leq_{2}, R_{2}\right\rangle$ depicted as

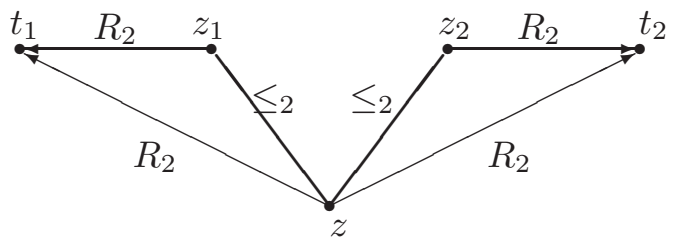

and put $\mu_{2}=\left\langle\mathcal{W}_{2}, \mathbf{v}_{2}\right\rangle$, where $\mathbf{v}_{2}(p)=\left\{t_{1}\right\}$ and $\mathbf{v}_{2}(q)=\left\{t_{2}\right\}$. It can be showed routinely that $\mu, z \not \models \nabla(p \wedge q) \rightarrow \nabla p \vee \nabla q=(\boxminus 2)^{\nabla}$, whence $(\boxminus 2)^{\nabla} \notin H K \square$ and $\nabla$ does not behave classically over Int. Again it can be shown nevertheless that $(\boxminus 1)^{\nabla}$ is a theorem of $H K \square$ and $H K \square$ is closed under contraposition rule for $\neg \square$. All the remaining cases can be considered the same way using the frame $\mathcal{W}_{2}$.

Corollary 2. Suppose $L \in$ NExt $H K \delta$, then

1. $\nabla \in\{\neg \diamond \neg, \neg \boxminus, \diamond \neg\}$ behaves classically in $L$ iff $(\square 2)^{\nabla}=\nabla p \wedge \nabla q \rightarrow$ $\nabla(p \wedge q) \in L$

2. $\nabla \in\{\neg \square \neg, \neg \vartheta, \boxminus \neg\}$ behaves classically in $L$ iff $(\diamond 2)^{\nabla}=\nabla(p \vee q) \rightarrow$ $\nabla p \vee \nabla q \in L$

3. $\nabla \in\{\neg \boxminus \neg, \neg \diamond, \square \neg\}$ behaves classically in $L$ iff $(\diamond 2)^{\nabla}=\nabla p \wedge \nabla q \rightarrow$ $\nabla(p \vee q) \in L$

4. $\nabla \in\{\neg \diamond \neg, \neg \square, \diamond \neg\}$ behaves classically in $L$ iff $(\boxminus 2)^{\nabla}=\nabla(p \wedge q) \rightarrow$ $\nabla p \vee \nabla q \in L$.

Let us point out an interesting distinction between those basic compositions which behave classically over Int and those that do not. Naturally, validity condition of a formula $\nabla \varphi$ for a basic composition $\nabla$ on worlds of corresponding models can be regarded as a first order formula. More formally we can consider a natural generalization of the standard translation $S T_{x}$ of propositional modal formulas into first order language (definition of standard translation for classical modal logics with $\square$ and $\diamond$ can be found in [1] and for Heyting-Kripke logics with negative modalities in [8]). For instance a first order formula

$$
S T_{x}(\neg \diamond \varphi)=\forall y\left(O(x, y) \rightarrow \neg \exists z\left(R(y, z) \wedge S T_{z}(\varphi)\right)\right)
$$

corresponds to composition $\neg \diamond$, where $O$ and $R$ are binary relations corresponding to ordering relation and accessibility relation, respectively, 
and all connectives on the right-hand side are classical. We can then transform this formula $S T_{x}(\nabla \varphi)$ into a prenex normal form. It turns out that basic compositions which behave classically over Int are exactly those whose corresponding first order formulas in prenex normal form do not have quantifier alternations.

We need some additional preliminary results.

For a $H K \delta$-frame $\mathcal{W}=\langle W, \leq, R\rangle$ by a maximal element $x \in W$ we will mean the one which is maximal wrt $\leq$. Let us abbreviate "for all maximal $x$ " as $\forall^{m} x$ and "exists maximal $x$ " as $\exists^{m} x$.

Proposition 3. Any canonical logic $L \in$ NExt $H K \delta$ is complete wrt a class of $H K \delta$-frames $\mathcal{W}=\langle W, \leq, R\rangle$, satisfying the following condition

$$
\forall x \in W \exists^{m} y \in W: x \leq y .
$$

Proof. It will suffice to show that the canonical $L$-frame $\mathcal{W}^{c}=\left\langle W^{c}\right.$, $\left.\subseteq, R^{c}\right\rangle$ satisfies (1).

Let $\Gamma$ be a prime $L$-theory. Consider a set $X:=\left\{\Gamma^{\prime} \in W^{c} \mid \Gamma \subseteq \Gamma^{\prime}\right\}$. It is clear that every chain in $X$ has an upper bound, whence by Zorn lemma there is a prime $L$-theory $\Delta$ such that $\Delta$ is a maximal element of $\mathcal{W}^{c}$ and $\Gamma \subseteq \Delta$.

Hereafter by $H K \delta$-frames we will understand frames satisfying (1).

Notice that for an arbitrary $H K \delta$-model $\mu=\langle W, \leq, R, \mathbf{v}\rangle, x \in W$ and formula $\varphi$ from (1) it follows that

$$
\mu, x \vDash \neg \neg \varphi \Longleftrightarrow \forall^{m} y \geq x \mu, y \vDash \varphi .
$$

Suppose $\mathcal{W}=\langle W, \leq, R\rangle$ is an $H K \delta$-frame. For $x, y \in W$ put

$$
x \bar{R} y \Longleftrightarrow \begin{cases}\exists z \in W(x R z \text { and } z \leq y) & \text { in case } \delta \in\{\square, \boxminus\} ; \\ \exists z \in W(x R z \text { and } y \leq z) & \text { in case } \delta \in\{\oslash, \nabla\} .\end{cases}
$$

It was shown in [2] and [4] that $H K \delta$ is complete wrt to a class of so called condensed frames in which $R=\bar{R}$. For our purposes it will suffice to notice, that for any $H K \delta$-model $\mu=\langle W, \leq, R, \mathbf{v}\rangle, x \in W$ and formula $\varphi$ the following holds:

$$
\begin{aligned}
& \mu, x \vDash \square \varphi \Longleftrightarrow \forall y \in W(x \bar{R} y \Rightarrow \mu, y \vDash \varphi) ; \\
& \mu, x \vDash \diamond \varphi \Longleftrightarrow \exists y \in W(x \bar{R} y \text { and } \mu, y \vDash \varphi) ; \\
& \mu, x \vDash \nabla \varphi \Longleftrightarrow \forall y \in W(x \bar{R} y \Rightarrow \mu, y \not \models \varphi) ; \\
& \mu, x \vDash \boxminus \varphi \Longleftrightarrow \exists y \in W(x \bar{R} y \text { and } \mu, y \not \models \varphi) .
\end{aligned}
$$


The next four sections are dedicated to axiomatizing those basic compositions, which behave classically over Int, excluding the case of composition $\neg \boxminus$ which was considered previously in [7].

\section{Basic compositions over intuitionistic logic}

\subsection{Composition $\neg \diamond$ as an impossibility operator}

In the following section we will obtain an axiomatization of composition $\neg \diamond$ of logic $H K \diamond$ as an impossibility operator.

We will prove that the following logic forms the above mentioned axiomatization

$$
H K(\neg \diamond):=H K \diamond+\neg \neg \diamond p \rightarrow \diamond p .
$$

We will start by identifying a class of $H K \diamond$-frames wrt which $H K(\neg \diamond)$ is complete.

Proposition 4. For an arbitrary $H K \diamond$-frame $\mathcal{W}=\langle W, \leq, R\rangle$ we have $\mathcal{W} \vDash \neg \neg \nabla p \rightarrow \forall p$ iff

$$
\forall x, y \in W\left(x R y \Rightarrow \exists^{m} z \geq x: z \bar{R} y\right) .
$$

Proof. $(\Longleftarrow)$ can be proved routinely.

$(\Longrightarrow)$. Suppose (3) fails, then for some $x, y \in W$ we have $x R y$ but $z \bar{R} y$ does not hold for every maximal $z \geq x$. Consider a valuation $\mathbf{v}$ such that $\mathbf{v}(p)=\{t \in W \mid t \geq y\}$ and put $\mu=\langle\mathcal{W}, \mathbf{v}\rangle$.

From the definition it follows that $\mu, x \not \models \forall p$, since $x R y$ and $y \in \mathbf{v}(p)$. Suppose now $z \geq x$ is maximal and $z R t$. If $t \geq y$ then $z \bar{R} y$ by definition, which contradicts our assumption. Therefore $t \nsupseteq y$, thus $\mu, t \not \models p$ and $\mu, z \vDash \nabla p$. Then by (2) we have $\mu, x \vDash \neg \neg \nabla p$, which leads to $\mu, x \not \models$ $\neg \neg \vee p \rightarrow \forall p$ and consequently to $\mathcal{W} \not \models \neg \neg \nabla p \rightarrow \diamond p$.

Theorem 3. Logic HK( $\neg \diamond)$ is canonical.

Proof. It will suffice to show that (3) holds for the canonical $H K(\neg \diamond)$ frame $\mathcal{W}^{c}=\left\langle W^{c}, \subseteq, R^{c}\right\rangle$. Firstly notice that in $\mathcal{W}^{c}$ we have $R^{c}=\overline{R^{c}}$ by definition.

Suppose $\Gamma R^{c} \Delta$ holds for $\Gamma, \Delta \in W^{c}$. Then consider a set $X=$ $\{\neg \nabla \varphi \mid \varphi \in \Delta\}$ and assume that $\Gamma \cup X \vdash \perp$ (we omit prefix $H K(\neg \diamond)$ ). Thus there are $\neg \nabla \varphi_{1}, \ldots, \neg \nabla \varphi_{n} \in X$ such that $\Gamma, \neg \nabla \varphi_{1}, \ldots, \neg \nabla \varphi_{n} \vdash$ 
$\perp$, from which using deduction theorem, the fact that $\Gamma R^{c} \Delta$ and theorems of logic $H K(\neg \diamond)$ we can infer the following sequence, with the new axiom of $H K(\neg \diamond)$ being used on the second to last step:

$$
\begin{gathered}
\Gamma \vdash \neg\left(\neg \nabla \varphi_{1} \wedge \cdots \wedge \neg \nabla \varphi_{n}\right) \Longrightarrow \Gamma \vdash \neg \neg\left(\nabla \varphi_{1} \vee \cdots \vee \nabla \varphi_{n}\right) \Longrightarrow \\
\Gamma \vdash \neg \neg \nabla\left(\varphi_{1} \wedge \cdots \wedge \varphi_{n}\right) \Longrightarrow \Gamma \vdash \vartheta\left(\varphi_{1} \wedge \cdots \wedge \varphi_{n}\right) \Longrightarrow \Delta \nvdash \varphi_{1} \wedge \cdots \wedge \varphi_{n} .
\end{gathered}
$$

On the other hand, from $\varphi_{1}, \ldots, \varphi_{n} \in \Delta$ we obtain $\varphi_{1} \wedge \cdots \wedge \varphi_{n} \in$ $\Delta$, which leads to a contradiction. By extension lemma there exists a prime $H K(\neg \diamond)$-theory $\Gamma^{\prime} \supseteq \Gamma \cup X$ and by (1) we have a maximal prime $H K(\neg \diamond)$-theory $\Gamma^{\prime \prime} \supseteq \Gamma^{\prime}$.

Suppose now $\varphi \in \Delta$, then $\neg \nabla \varphi \in X \subseteq \Gamma^{\prime \prime}$ and $\nabla \varphi \notin \Gamma^{\prime \prime}$ since $\Gamma^{\prime \prime}$ is not trivial by definition. Thus we have $\Gamma^{\prime \prime} R^{c} \Delta$, which means (3) does hold in $\mathcal{W}^{c}$.

Theorem 4. Put $\nabla=\neg \diamond$. Then for any formula $\varphi \in$ For $\mathcal{L} \diamond$ we have

$$
\varphi \in H K(\neg \diamond) \Longleftrightarrow(\varphi)^{\nabla} \in H K \diamond .
$$

Proof. $(\Longrightarrow)$. It will suffice to show that $(\neg \neg \diamond p \rightarrow \diamond p)^{\nabla}=\neg \neg \neg \diamond p$ $\rightarrow \neg \diamond p \in H K \diamond$ which follows immediately from $\neg p \leftrightarrow \neg \neg \neg p \in$ Int.

$(\Longleftarrow)$. Suppose $\varphi \notin H K(\neg \diamond)$ then there is an $H K(\neg \diamond)$-model $\mu=$ $\langle W, \leq, R, \mathbf{v}\rangle$ and $x_{0} \in W$ such that $\mu, x_{0} \not \models \varphi$.

Consider $\mu^{\prime}=\left\langle W, \leq, R^{\prime}, \mathbf{v}\right\rangle$, where for all $x, y \in W$

$$
x R^{\prime} y \Longleftrightarrow x \bar{R} y \text { and } x \text { is maximal. }
$$

Let us show that $\mu^{\prime}$ is an $H K \diamond$-model, by proving that the interplay condition between $R^{\prime}$ and $\leq$ holds. Assume $x \leq y$ and $x R^{\prime} z$, then by definition of $R^{\prime}$ if follows that $x$ is maximal, hence $x=y$ and thus $y R^{\prime} z$.

Next we will show by induction on complexity of formula $\psi$ that for any $x \in W$ we have

$$
\mu, x \vDash \psi \Longleftrightarrow \mu^{\prime}, x \vDash(\psi)^{\nabla} .
$$

All non-modal cases are trivial since $\mu$ and $\mu^{\prime}$ share the same $W, \leq$, and $\mathbf{v}$.

Suppose $\mu, x \not \models \diamond \psi$, then there is $y \in W$ such that $x R y$ and $\mu, y \vDash \psi$. By (3) there is a maximal $z \geq x$ such that $z \bar{R} y$. Then we have $z R^{\prime} y$ by definition and by induction hypothesis we obtain $\mu^{\prime}, y \vDash(\psi)^{\nabla}$. Thus $\mu^{\prime}, z \vDash \diamond(\psi)^{\nabla}$ hence $\mu^{\prime}, x \not \models \neg \diamond(\psi)^{\nabla}=(\diamond \psi)^{\nabla}$. 
Now let $\mu^{\prime}, x \not \models(\nabla \psi)^{\nabla}=\neg \diamond(\psi)^{\nabla}$. Then there are $y, z \in W$ such that $y \geq x, y R^{\prime} z$ and $\mu^{\prime}, z \vDash(\psi)^{\nabla}$. From definition of $R^{\prime}$ we infer that $y$ is maximal and $y \bar{R} z$. By induction hypothesis we obtain $\mu, z \vDash \psi$, hence $\mu, y \not \models \diamond \psi$ and $\mu, x \not \models \diamond \psi$ by the generalized heredity condition.

The proof by induction is completed.

Finally from $\mu, x_{0} \not \models \varphi$ we infer $\mu^{\prime}, x_{0} \not \models(\varphi)^{\nabla}$ and thus $(\varphi)^{\nabla} \notin H K \diamond$.

The last theorem essentially shows that $H K(\neg \diamond)$ coincides with the $\langle\wedge, \vee, \rightarrow, \neg, \neg \diamond\rangle$-fragment of logic $H K \diamond$ in which $\neg \diamond$ is denoted as $\diamond$.

\subsection{Composition $\square \neg$ as an impossibility operator}

We will show that logic

$$
H K(\square \neg):=H K \diamond+\diamond p \rightarrow \diamond \neg \neg p
$$

constitutes an axiomatization of composition $\square \neg$ in logic $H K \square$ as an impossibility operator.

The scheme will follow the one we developed in the previous section.

Proposition 5. Let $\mathcal{W}=\langle W, \leq, R\rangle$ be an $H K \diamond$-frame. Then $\mathcal{W} \vDash$ $\diamond p \rightarrow \diamond \neg \neg p$ holds iff

$$
\forall x, y \in W\left(x R y \Rightarrow \exists^{m} z \geq y: x R z\right) .
$$

Proof. $(\Longleftarrow)$ can be proved routinely.

$(\Longrightarrow)$. Suppose there are $x, y \in W$ such that $x R y$ but for every maximal $z \geq y$ the condition $x R z$ does not hold. Let us define a valuation $\mathbf{v}$ such that for any $t \in W$

$$
t \in \mathbf{v}(p) \Longleftrightarrow \nexists z \in W(z \geq t \text { and } x R z) .
$$

and consider $\mu=\langle\mathcal{W}, \mathbf{v}\rangle$.

If $z \in W$ then $x R z$ implies $z \notin \mathbf{v}(p)$ by definition, hence $\mu, x \vDash \vee p$. Assume $t \geq y$ is maximal then clearly there is no $t^{\prime} \geq t$ such that $x R t^{\prime}$, thus $t \in \mathbf{v}(p)$ and $\mu, t \vDash p$. Then by (2) we have $\mu, y \vDash \neg \neg p$, hence $\mu, x \not \models \diamond \neg \neg p$. Therefore $\mu, x \not \models \diamond p \rightarrow \diamond \neg \neg p$ and $\mathcal{W} \not \models \diamond p \rightarrow \diamond \neg \neg p$. $\dashv$

Theorem 5. HK( $\square \neg)$ is a canonical logic. 
Proof. We show that the canonical $H K(\square \neg)$-frame $\mathcal{W}^{c}=\left\langle W^{c}, \subseteq, R^{c}\right\rangle$ satisfies (4).

Suppose $\Gamma R^{c} \Delta$ for some $\Gamma, \Delta \in W^{c}$. Consider $X:=\{\neg \varphi \mid \nabla \varphi \in \Gamma\}$ and assume that $\Delta \cup X \vdash \perp$. Then there are formulas $\neg \varphi_{1}, \ldots, \neg \varphi_{n} \in X$ such that $\Delta, \neg \varphi_{1}, \ldots, \neg \varphi_{n} \vdash \perp$. Using $\Gamma R^{c} \Delta$ and theorems of logic $H K(\square \neg)$ it is not hard to obtain the following sequence of inferences:

$$
\Delta \vdash \neg\left(\neg \varphi_{1} \wedge \cdots \wedge \neg \varphi_{n}\right) \Longrightarrow \Delta \vdash \neg \neg\left(\varphi_{1} \vee \cdots \vee \varphi_{n}\right) \Longrightarrow
$$

$\Gamma \nvdash \diamond \neg \neg\left(\varphi_{1} \vee \cdots \vee \varphi_{n}\right) \Longrightarrow \Gamma \nvdash \vartheta\left(\varphi_{1} \vee \cdots \vee \varphi_{n}\right) \Longrightarrow \Gamma \nvdash \nabla \varphi_{1} \wedge \cdots \wedge \vartheta \varphi_{n}$.

Thus we have $\nabla \varphi_{1} \wedge \cdots \wedge \nabla \varphi_{n} \notin \Gamma$. On the other hand from $\nabla \varphi_{1}, \ldots, \nabla \varphi_{n} \in \Gamma$ we can infer $\nabla \varphi_{1} \wedge \cdots \wedge \nabla \varphi_{n} \in \Gamma$, which leads to a contradiction. Therefore we have $\Delta \cup X \nvdash \perp$, then by extension lemma there is a prime $H K(\square \neg)$-theory $\Delta^{\prime} \supseteq \Delta \cup X$ and by (1) there is a maximal $\Delta^{\prime \prime} \in W^{c}$ such that $\Delta^{\prime \prime} \supseteq \Delta^{\prime}$.

Suppose $\nabla \varphi \in \Gamma$ then $\neg \varphi \in X \subseteq \Delta^{\prime \prime}$, hence $\varphi \notin \Delta^{\prime \prime}$. Consequently we have $\Gamma R^{c} \Delta^{\prime \prime}$ and (4) is satisfied.

Theorem 6. Let $\nabla=\square \neg$, then for any formula $\varphi \in$ For $\mathcal{L} \diamond$ we have

$$
\varphi \in H K(\square \neg) \Longleftrightarrow(\varphi)^{\nabla} \in H K \square .
$$

Proof. ( $\Longrightarrow)$ is trivial since $\neg p \leftrightarrow \neg \neg \neg p \in$ Int.

$(\Longleftarrow)$. Suppose $\varphi \notin H K(\square \neg)$ then there is an $H K(\square \neg)$-model $\mu=$ $\langle W, \leq, R, \mathbf{v}\rangle$ and $x_{0} \in W$ such that $\mu, x_{0} \not \models \varphi$.

Consider $\mu^{\prime}=\left\langle W, \leq, R^{\prime}, v\right\rangle$, where

$$
x R^{\prime} y \Longleftrightarrow x R y \text { and } y \text { is maximal. }
$$

Assume that $x \leq y$ and $y R^{\prime} z$ for some $x, y, z \in W$. Then by definition $z$ is maximal and $y R z$ thus by the interplay condition between $\leq$ and $R$ we have $x R z$ and consequently $x R^{\prime} z$. Therefore $\mu^{\prime}$ is an $H K \square$-model.

We show by induction on complexity of $\psi$ that for all $x \in W$

$$
\mu, x \vDash \psi \Longleftrightarrow \mu^{\prime}, x \vDash(\psi)^{\nabla} .
$$

We consider only the modal case.

Suppose $\mu, x \not \models \nabla \psi$ then there is $y \in W$ such that $x R y$ and $\mu, y \vDash \psi$. By (4) there is a maximal $z \geq x$ such that $x R z$. By the generalized heredity condition we obtain $\mu, z \vDash \psi$ and by induction hypothesis we 
have $\mu^{\prime}, z \vDash(\psi)^{\nabla}$ hence $\mu^{\prime}, z \not \models \neg(\psi)^{\nabla}$. Moreover it is clear that $x R^{\prime} z$, thus $\mu^{\prime}, x \not \models \square \neg(\psi)^{\nabla}=(\nabla \psi)^{\nabla}$.

Suppose now $\mu^{\prime}, x \not \models(\nabla \psi)^{\nabla}=\square \neg(\psi)^{\nabla}$. Then there are $y, z \in W$ such that $x R^{\prime} y, z \geq y$ and $\mu^{\prime}, z \vDash(\psi)^{\nabla}$. Then $y$ is maximal by definition of $R^{\prime}$, hence $y=z$ and $x R z$. By induction hypothesis we also have $\mu, z \vDash \psi$ thus $\mu, z \not \models \nabla \psi$, which completes the proof by induction.

Finally from $\mu, x_{0} \not \models \varphi$ we infer $\mu^{\prime}, x_{0} \not \models(\varphi)^{\nabla}$ and $(\varphi)^{\nabla} \notin H K \square . \quad \dashv$

\subsection{Composition $\neg \boxminus \neg$ as an impossibility operator}

We will obtain an axiomatization of composition $\neg \boxminus \neg$ in $H K \boxminus$ as an impossibility operator in the form of logic

$$
H K(\neg \boxminus \neg):=H K \diamond+\neg \neg \diamond p \rightarrow \diamond p+\ominus p \rightarrow \diamond \neg \neg p .
$$

It follows directly from Propositions 4 and 5 that any $H K(\neg \boxminus \neg)$ frame satisfies conditions (3) and (4). Then by combining proofs of Theorems 3 and 5 it can be easily shown that $H K(\neg \boxminus \neg)$ is canonical, from which we infer that $H K(\neg \boxminus \neg)$ is complete wrt the class of all $H K \diamond$-frames satisfying (3) and (4). Moreover, for any $H K(\neg \boxminus \neg)$-frame $\mathcal{W}=\langle W, \leq, R\rangle$ we clearly have

$$
\forall x, y \in W\left(x R y \Rightarrow \exists^{m} z \geq x \exists^{m} t \geq y: z \bar{R} t\right) .
$$

Theorem 7. Let $\nabla=\neg \boxminus \neg$ then for any formula $\varphi \in$ For $\mathcal{L} \vartheta$

$$
\varphi \in H K(\neg \boxminus \neg) \Longleftrightarrow(\varphi)^{\nabla} \in H K \boxminus .
$$

Proof. Let us prove $(\Longleftarrow)$. If $\varphi \notin H K(\neg \boxminus \neg)$ then there is an $H K(\neg \boxminus \neg)$-model $\mu=\langle W, \leq, R, \mathbf{v}\rangle$ and $x_{0} \in W$ such that $\mu, x_{0} \not \models \varphi$.

It is easy to show that $\mu^{\prime}=\left\langle W, \leq, R^{\prime}, \mathbf{v}\right\rangle$, where for all $x, y \in W$

$$
x R^{\prime} y \Longleftrightarrow x \bar{R} y \text { and } x, y \text { are maximal, }
$$

is an $H K \boxminus$-model.

Again we show by induction on complexity of $\psi$ that for $x \in W$

$$
\mu, x \vDash \psi \Longleftrightarrow \mu^{\prime}, x \vDash(\psi)^{\nabla}
$$

and consider only the modal case. 
Suppose $\mu, x \not \models \nabla \psi$, then there is $y \in W$ such that $x R y$ and $\mu, y \vDash \psi$. By (5) we have a maximal $z \geq x$ and a maximal $t \geq z \operatorname{such}$ that $z \bar{R} t$. Thus $\mu, t \vDash \psi$ from which by induction hypothesis we infer $\mu^{\prime}, t \vDash(\psi)^{\nabla}$ hence $\mu^{\prime}, t \not \models \neg(\psi)^{\nabla}$. Now by definition of $R^{\prime}$ we have $z R^{\prime} t$, thus $\mu^{\prime}, z \vDash$ $\boxminus \neg(\psi)^{\nabla}$. Finally we have $\mu^{\prime}, z \not \models \neg \boxminus \neg(\psi)^{\nabla}$ and by the generalized heredity condition we obtain $\mu^{\prime}, x \not \models(\diamond \psi)^{\nabla}$.

If $\mu^{\prime}, x \not \models \neg \boxminus \neg(\psi)^{\nabla}$ then there are $y, z, t \in W$ such that $y \geq x, y R^{\prime} z$, $t \geq z$ and $\mu^{\prime}, t \vDash(\psi)^{\nabla}$. From $y R^{\prime} z$ we infer that $y$ and $z$ are maximal and $y \bar{R} z$. Thus $z=t$ and $y \bar{R} t$. By induction hypothesis we have $\mu, t \vDash \psi$, hence $\mu, y \not \models \nabla \psi$ and by the generalized heredity condition $\mu, x \not \models \nabla \psi$.

Finally, from $\mu, x_{0} \not \models \varphi$ we infer $\mu^{\prime}, x_{0} \not \models(\varphi)^{\nabla}$, thus $(\varphi)^{\nabla} \notin H K \boxminus . \quad \dashv$

\subsection{Composition $\boxminus \neg$ as a possibility operator}

We will show that logic

$$
H K(\boxminus \neg):=H K \diamond+\diamond \neg \neg p \rightarrow \diamond p
$$

constitutes an axiomatization of composition $\boxminus \neg$ in $H K \boxminus$ as a possibility operator.

Proposition 6. For an $H K \diamond$-frame $\mathcal{W}=\langle W, \leq, R\rangle$ we have $\mathcal{W} \vDash$ $\diamond \neg \neg p \rightarrow \diamond p$ iff (4) holds (see Section 2.2).

Proof. Again we prove only $(\Longrightarrow)$. Suppose $x R y$ for some $x, y \in W$ yet for any maximal $z \geq y$ condition $x R z$ fails. It is easy to show that $\mu=\langle\mathcal{W}, \mathbf{v}\rangle$, where for all $t \in W$

$$
t \in \mathbf{v}(p) \Longleftrightarrow t \geq y \text { and } t \text { maximal, }
$$

is an $H K \diamond$-model. Moreover from the definition it follows directly that $\mu, x \vDash \diamond \neg \neg p$. On the other hand let $x R z$, then $z$ is not a maximal world such that $z \geq y$, thus $z \notin \mathbf{v}(p)$ and $\mu, z \not \models p$. Therefore, $\mu, x \not \models \diamond p$ hence $\mu, x \nvdash \bullet \neg \neg p \rightarrow \diamond p$ and $\mathcal{W} \not \models \diamond \neg \neg p \rightarrow \diamond p$.

TheOREM 8. Logic HK( $\boxminus \neg)$ is canonical.

Proof. We show that (4) holds for the canonical $H K(\boxminus \neg)$-frame $\mathcal{W}^{c}=$ $\left\langle W^{c}, \subseteq, R^{c}\right\rangle$.

Suppose for some $\Gamma, \Delta \in W^{c}$ we have $\Gamma R^{c} \Delta$. Consider $X:=\{\neg \varphi \mid$ $\diamond \varphi \notin \Gamma\}$ and assume $\Delta \cup X \vdash \perp$. Then there are $\neg \varphi_{1}, \ldots, \neg \varphi_{n} \in X$ 
such that $\Delta, \neg \varphi_{1}, \ldots, \neg \varphi_{n} \vdash \perp$, from which the following sequence of inferences follows

$$
\begin{gathered}
\Delta \vdash \neg\left(\neg \varphi_{1} \wedge \cdots \wedge \neg \varphi_{n}\right) \Longrightarrow \Delta \vdash \neg \neg\left(\varphi_{1} \vee \cdots \vee \varphi_{n}\right) \Longrightarrow \\
\Gamma \vdash \diamond \neg \neg\left(\varphi_{1} \vee \cdots \vee \varphi_{n}\right) \Longrightarrow \Gamma \vdash \diamond\left(\varphi_{1} \vee \cdots \vee \varphi_{n}\right) \Longrightarrow \Gamma \vdash \diamond \varphi_{1} \vee \cdots \vee \diamond \varphi_{n} .
\end{gathered}
$$

Then by disjunctive property we obtain $\diamond \varphi_{i} \in \Gamma$ for some $i$, which contradicts the definition of $X$. Thus by extension lemma and (1) we have $\Delta^{\prime} \in W^{c}$ such that $\Delta^{\prime}$ is maximal and $\Delta^{\prime} \supseteq \Delta \cup X$. Suppose now $\diamond \varphi \notin \Gamma$ thus $\neg \varphi \in X \subseteq \Delta^{\prime}$ and $\varphi \notin \Delta^{\prime}$. Therefore $\Gamma R^{c} \Delta^{\prime}$ and (4) does hold.

Theorem 9. If $\nabla=\boxminus \neg$ then for any formula $\varphi \in$ For $\mathcal{L} \diamond$ the following equivalence holds:

$$
\varphi \in H K(\boxminus \neg) \Longleftrightarrow(\varphi)^{\nabla} \in H K \boxminus .
$$

Proof. We prove $(\Longleftarrow)$. Suppose $\varphi \notin H K(\boxminus \neg)$ then there is an $H K(\boxminus \neg)$-model $\mu=\langle W, \leq, R, \mathbf{v}\rangle$ and $x_{0} \in W$ such that $\mu, x_{0} \not \models \varphi$.

Consider $\mu^{\prime}=\left\langle W, \leq, R^{\prime}, \mathbf{v}\right\rangle$, where

$$
x R^{\prime} y \Longleftrightarrow x R y \text { and } y \text { is maximal. }
$$

Now, suppose $x \leq y$ and $x R^{\prime} z$ for $x, y, z \in W$, then $z$ is maximal by definition of $R^{\prime}$ and $x R z$, from which we infer $y R z$ by interplay condition for $R$, thus having $y R^{\prime} z$. Consequently $\mu^{\prime}$ is an $H K \boxminus$-model.

By induction on complexity of $\psi$ we show that for any $x \in W$

$$
\mu, x \vDash \psi \Longleftrightarrow \mu^{\prime}, x \vDash(\psi)^{\nabla} .
$$

Once again we consider only the modal case.

Suppose $\mu, x \vDash \diamond \psi$ then there is $y \in W$ such that $x R y$ and $\mu, y \vDash \psi$. By (4) there is also a maximal $z \geq x$ such that $x R z$. By generalized heredity condition we have $\mu, z \vDash \psi$, and by induction hypothesis $\mu^{\prime}, z \vDash$ $(\psi)^{\nabla}$, hence $\mu^{\prime}, z \not \models \neg(\psi)^{\nabla}$. Moreover it is clear that $x R^{\prime} z$, thus $\mu^{\prime}, x \vDash$ $\boxminus \neg(\psi)^{\nabla}=(\diamond \psi)^{\nabla}$.

Suppose $\mu^{\prime}, x \vDash(\diamond \psi)^{\nabla}=\boxminus \neg(\psi)^{\nabla}$. Then there are $y, z \in W$ such that $x R^{\prime} y, z \geq y$ and $\mu^{\prime}, z \vDash(\psi)^{\nabla}$. Since $y$ is maximal by definition of $R^{\prime}$, we have $y=z$ and $x R z$. By induction hypothesis we obtain $\mu, z \vDash \psi$, thus $\mu, z \vDash \diamond \psi$, which completes the proof by induction.

From $\mu, x_{0} \not \models \varphi$ using the result above we infer $\mu^{\prime}, x_{0} \not \models(\varphi)^{\nabla}$, which leads to $(\varphi)^{\nabla} \notin H K \boxminus$. 


\subsection{Where everything behaves}

We have obtained axiomatizations of all basic compositions which behave classically over Int. Notice that all of them are axiomatized by quite similar formulas, which essentially stem from the following fact: we have $\neg \varphi \leftrightarrow \neg \neg \neg \varphi \in$ Int, thus naturally for a basic composition $\nabla=\neg \delta$ we have $\nabla \varphi \leftrightarrow \neg \neg \nabla \varphi \in H K \delta$ and for a basic composition $\nabla=\delta \neg$ we have $\nabla \varphi \leftrightarrow \nabla \neg \neg \varphi \in H K \delta$. For $\nabla=\neg \delta \neg$ we have both these conditions.

A natural question arises on what properties intuitionistic logic lacks which would allow all basic compositions to behave classically over it. It turns out that $K C$ constitutes the smallest superintuitionistic logics in which all basic compositions behave classically. We remind the reader, that $K C$ can be represented as

$$
K C=\operatorname{Int}+\neg(p \wedge q) \rightarrow \neg p \vee \neg q
$$

making it the smallest superintuitionistic logic in which all De Morgan laws hold (this follows from a result proved in [16]).

Lemma 3. Suppose $\mathfrak{A}=\langle A, \vee, \wedge, \rightarrow, \neg, 0,1\rangle$ is a Heyting algebra. Then

1. $\mathfrak{A}_{1}=\langle A, \vee, \wedge, \rightarrow, \neg, \square, 0,1\rangle$, where $\square a=a$ for all $a \in A$, is a $\square$ algebra;

2. $\mathfrak{A}_{2}=\langle A, \vee, \wedge, \rightarrow, \neg, \diamond, 0,1\rangle$, where $\diamond a=a$ for all $a \in A$, is a $\diamond$ algebra;

3. $\mathfrak{A}_{3}=\langle A, \vee, \wedge, \rightarrow, \neg, \diamond, 0,1\rangle$, where $\nabla a=\neg a$ for all $a \in A$, is a $\vartheta$ algebra;

4. if $b \in A$, then $\mathfrak{A}_{4}=\langle A, \vee, \wedge, \rightarrow, \neg, \diamond, 0,1\rangle$, where for $a \in A$ we have

$$
\diamond a= \begin{cases}0, & \text { if } a \leq b ; \\ 1, & \text { otherwise }\end{cases}
$$

is a $\diamond$-algebra;

5. if $b \in A$, then $\mathfrak{A}_{5}=\langle A, \vee, \wedge, \rightarrow, \neg, \vartheta, 0,1\rangle$, where for $a \in A$ we have

$$
\nabla a= \begin{cases}1, & \text { if } a \leq b ; \\ 0, & \text { otherwise }\end{cases}
$$

is a $\diamond$-algebra.

Proof. 1. It is clear that in $\mathfrak{A}_{1}$ we have $\square 1=1$ and for all $a, b \in A$ we have $\square(a \wedge b)=a \wedge b=\square a \wedge \square b$. 
2. In $\mathfrak{A}_{2}$ we have $0=\diamond 0$ and $\diamond a \vee \diamond b=a \vee b=\diamond(a \vee b)$ holds for all $a, b \in A$.

3. In $\mathfrak{A}_{3}$ we have $\nabla 0=\neg 0=1$ and since $\neg(\varphi \vee \psi) \leftrightarrow \neg \varphi \wedge \neg \psi$ is an intuitionistic theorem for all $a, b \in A$ we obtain $\diamond(a \vee b)=\neg(a \vee b)=$ $\neg a \wedge \neg b=\diamond a \wedge \diamond b$.

4. Since $0 \leq b$ we have $\diamond 0=0$. Consider arbitrary $c, d \in A$. Suppose $c \not \leq b$, then clearly $c \vee d \not \leq b$, hence $\diamond(c \vee d)=1=1 \vee \diamond d=\diamond c \vee \diamond d$. Similarly in case $d \not \leq b$. Suppose now $c \leq b$ and $d \leq b$, then $c \vee d \leq b$ thus $\diamond c \vee \diamond d=0 \vee 0=0=\diamond(c \vee d)$.

5. From $0 \leq b$ we immediately infer $\diamond 0=1$. Suppose $c, d \in A$. If $c \not \leq b$ then $c \vee d \not \leq b$ thus $\nabla(c \vee d)=0=0 \wedge \nabla d=\nabla c \wedge \nabla d$. Similarly in case $d \not \leq b$. If $c \leq b$ and $d \leq b$ then $c \vee d \leq b$ and therefore $\nabla c \wedge \nabla d=$ $1 \wedge 1=1=\diamond(c \vee d)$.

TheOREM 10. Let $\nabla$ be a basic composition such that $\nabla$ does not behave classically over Int (i.e. $\nabla \in\{\neg \square, \diamond \neg, \neg \vartheta, \diamond \neg, \neg \square \neg, \neg \diamond \neg, \neg \diamond \neg\}$ ). Then $K C$ is the smallest superintuitionistic logic such that $\nabla$ behaves classically over $\mathrm{KC}$.

Proof. Using De Morgan laws, modal axioms of Heyting-Kripke logics and Corollary 2 it is easy to show that all these basic compositions behave classically over $K C$. Consider for instance composition $\neg \square$. Since $\square p \wedge$ $\square q \rightarrow \square(p \wedge q) \in H K \square \subseteq K C \square$ then by contraposition for intuitionistic negation we have $\neg \square(p \wedge q) \rightarrow \neg(\square p \wedge \square q) \in K C \square$. Using De Morgan laws we also obtain $\neg(\square p \wedge \square q) \rightarrow \neg \square p \vee \neg \square q \in K C \square$, from which we infer $\neg \square(p \wedge q) \rightarrow \neg \square p \vee \neg \square q \in K C \square$, which means that $\neg \square$ behaves classically over $K C$ by Corollary 2 .

Consider a superintuitionistic logic $L$. We will assume that $K C \nsubseteq L$ and show that each of the above mentioned compositions $\nabla$ does not behave classically over $L$. From $K C \nsubseteq L$ it follows that $\neg(p \wedge q) \rightarrow$ $\neg p \vee \neg q \notin L$ and $\neg p \vee \neg \neg p \notin L$, hence there is a Heyting-algebra $\mathfrak{A}=$ $\langle A, \vee, \wedge, \rightarrow, \neg, 0,1\rangle$ such that $L \subseteq L \mathfrak{A}, \mathfrak{A} \not \models \neg(p \wedge q) \rightarrow \neg p \vee \neg q$ and $\mathfrak{A} \not \models \neg p \vee \neg \neg p$. From the last two conditions there exist $b, c, d \in A$ such that $\neg(b \wedge c) \rightarrow \neg b \vee \neg c \neq 1$ and $\neg d \vee \neg \neg d \neq 1$.

1. Suppose $\nabla=\neg \square$. By item 1 of Lemma 3 an algebra $\mathfrak{A}_{1}=$ $\langle A, \vee, \wedge, \rightarrow, \neg, \square, 0,1\rangle$, where $\square a=a$ for all $a \in A$, is a $\square$-algebra. Moreover it is clear that in $\mathfrak{A}_{1}$ the following holds

$$
1 \neq \neg(b \wedge c) \rightarrow \neg b \vee \neg c=\neg \square(b \wedge c) \rightarrow \neg \square b \vee \neg \square c
$$


hence $\mathfrak{A}_{1} \not \models \neg \square(p \wedge q) \rightarrow \neg \square p \vee \neg \square q$. It is easy to see that $L \square \subseteq L \mathfrak{A}_{1}$, thus $\neg \square(p \wedge q) \rightarrow \neg \square p \vee \neg \square q=(\boxminus 2)^{\nabla} \notin L \square$ by Theorem 2, from which using item 4 of Corollary 2 we infer that composition $\neg \square$ does not behave classically over $L$.

2. Let $\nabla=\neg \square \neg$. Firstly, since $\neg \neg(\neg p \vee p), \neg p \leftrightarrow \neg \neg \neg p \in$ Int, we have in $\mathfrak{A}$ :

$$
1 \neq \neg \neg(\neg d \vee d) \rightarrow \neg d \vee \neg \neg d=\neg \neg(\neg d \vee d) \rightarrow \neg \neg \neg d \vee \neg \neg d
$$

Then in algebra $\mathfrak{A}_{1}$ defined in the previous item we obtain

$$
1 \neq \neg \square \neg(\neg d \vee \neg \neg d) \rightarrow \neg \square \neg \neg d \vee \neg \square \neg d,
$$

thus $\neg \square \neg(\neg p \vee p) \rightarrow \neg \square \neg \neg p \vee \neg \square \neg p \notin L \square$. All that remains is to notice that the last formula can be obtained by substitution from formula $(\diamond 2)^{\nabla}$ hence by item 2 of Corollary 2 composition $\neg \square \neg$ does not behave classically over $L$.

3. Let $\nabla=\diamond \neg$. By item 2 of Lemma 3 algebra $\mathfrak{A}_{2}=\langle A, \vee, \wedge, \rightarrow$, $\neg, \diamond, 0,1\rangle$, where $\diamond a=a$ for all $a \in A$, is a $\diamond$-algebra. Then in $\mathfrak{A}_{2}$ we have

$$
1 \neq \neg(b \wedge c) \rightarrow \neg b \vee \neg c=\diamond \neg(b \wedge c) \rightarrow \diamond \neg b \vee \diamond \neg c .
$$

Thus $(\boxminus 2)^{\nabla} \notin L \diamond$, and composition $\diamond \neg$ does not behave classically over $L$ by item 4 of Corollary 2.

4. Suppose $\nabla=\neg \diamond \neg$. From $\neg(b \wedge c) \rightarrow \neg b \vee \neg c \neq 1$ we have $\neg(b \wedge c) \not \leq \neg b \vee \neg c$ in $\mathfrak{A}$. By item 4 of Lemma $3 \mathfrak{A}_{3}=\langle A, \vee, \wedge, \rightarrow$ $, \neg, \diamond, 0,1\rangle$, where for $a \in A$

$$
\diamond a= \begin{cases}0, & \text { if } a \leq \neg b \vee \neg c ; \\ 1, & \text { otherwise }\end{cases}
$$

is a $\diamond$-algebra. Then in $\mathfrak{A}_{3}$ we have $\diamond(\neg b \vee \neg c)=0$ and $\diamond \neg(b \wedge c)=1$, hence $1=\neg \diamond(\neg b \vee \neg c)=\neg(\diamond \neg b \vee \diamond \neg c)=\neg \diamond \neg b \wedge \neg \diamond \neg c \not \leq \neg \diamond \neg(b \wedge c)=0$. Thus $\neg \diamond \neg b \wedge \neg \diamond \neg c \rightarrow \neg \diamond \neg(b \wedge c) \neq 1$ in $\mathfrak{A}_{3}$ which leads to $(\square 2)^{\nabla} \notin L \diamond$ and so composition $\neg \diamond \neg$ does not behave classically over $L$ by item 1 of Corollary 2. 
5. Let $\nabla=\diamond \neg$. By item 5 of Lemma $3 \mathfrak{A}_{4}=\langle A, \vee, \wedge, \rightarrow, \neg, \diamond, 0,1\rangle$, where for all $a \in A$ we have

$$
\nabla a= \begin{cases}1, & \text { if } a \leq \neg c \vee \neg b ; \\ 0, & \text { otherwise, }\end{cases}
$$

is a $\diamond$-algebra. Then in $\mathfrak{A}_{4}$ the following holds:

$$
1=\vartheta(\neg c \vee \neg b)=\diamond \neg c \wedge \vartheta \neg b \not \leq \forall \neg(c \wedge b)=0 .
$$

Thus $\vartheta \neg c \wedge \vartheta \neg b \rightarrow \vartheta \neg(c \wedge b) \neq 1$, hence $(\square 2)^{\nabla} \notin L \ominus$ which means that composition $\vartheta \neg$ does not behave classically over $L$ by item 1 of Corollary 2.

6. Let $\nabla=\neg \vartheta$. By item 3 of Lemma $3 \mathfrak{A}_{5}=\langle A, \vee, \wedge, \rightarrow, \neg, \nabla, 0,1\rangle$, where for all $a \in A$ we have $\vartheta a=\neg a$, is a $\vartheta$-algebra. Then using (6) we obtain in $\mathfrak{A}_{5}$

$$
1 \neq \neg \neg(\neg d \vee d) \rightarrow \neg \neg \neg d \vee \neg \neg d=\neg \vartheta(\neg d \vee d) \rightarrow \neg \vartheta \neg d \vee \neg \vartheta d,
$$

hence $\neg \vartheta(\neg p \vee p) \rightarrow \neg \vartheta \neg p \vee \neg \vartheta p \notin L \vartheta$. Thus composition $\neg \vartheta$ does not behave classically over $L$ by item 2 of Corollary 2 and the fact that $\neg \vartheta(\neg p \vee p) \rightarrow \neg \vartheta \neg p \vee \neg \vartheta p$ can be obtain by substitution from formula $(\diamond 2)^{\nabla}$.

7. Finally, suppose $\nabla=\neg \diamond \neg$. Consider again $\mathfrak{A}_{5}$ from the previous item. We have in $\mathfrak{A}_{5}$

$$
\begin{aligned}
1 \neq \neg(b \wedge c) \rightarrow \neg b \vee \neg c=\neg \neg \neg(b \wedge c) & \rightarrow \neg \neg \neg b \vee \neg \neg \neg c= \\
& \neg \vartheta \neg(b \wedge c) \rightarrow \neg \vartheta \neg b \vee \neg \vartheta \neg c,
\end{aligned}
$$

thus $(\boxminus 2)^{\nabla} \notin L \ominus$ and composition $\neg \vartheta \neg$ does not behave classically over $L$ by item 4 of Corollary 2 .

Naturally, there remains an open question of axiomatizing all basic compositions over $K C$.

\section{References}

[1] Blackburn P., and J. van Benthem, "Modal logic: A semantic perspective", pages 1-84 in Handbook of Modal Logic. Volume 3, P. Blackburn, J. van Benthem, and F. Wolter (eds.), series "Studies in Logic and Practical Reasoning", Elsevier Science Inc., New York, 2006.

DOI: $10.1016 /$ S1570-2464(07)80004-8 
[2] Božić, M., and K. Došen, "Models for normal intuitionistic modal logics", Studia Logica 43 (1984): 217-245.

[3] Cabalar, P., S. P. Odintsov, and D. Pearce, "Logical foundations of wellfounded semantics", pages 25-36 in Principles of Knowledge Representation and Reasoning: Proceedings of the 10th International Conference (KR2006), P. Doherty et al (eds.), AAAI Press, Menlo Park, California, 2006.

[4] Došen, K., "Negative modal operators in intuitionistic logic", Publication de l'Instutute Mathematique, Nouv. Ser., 35 (1984): 3-14.

[5] Došen, K., "Models for stronger normal intuitionistic modal logics", Studia Logica 44 (1985): 39-70.

[6] Drobyshevich, S. A., "A double negation operator in logic $N^{*}$ ", Vestnik NSU, Series: Math., mech. and informatics, 13, 4 (2013): 68-83.

[7] Drobyshevich, S. A., "Composition of an intuitionistic negation and negative modalities as a necessity operator", Algebra and Logic, 52, 3 (2013): 305-331. DOI: 10.1007/s10469-013-9235-8

[8] Drobyshevich, S. A., and S. P. Odintsov, "Finite model property for negative modalities" (in Russian), Siberian Electronic Mathematical Reports, 10 (2013): 1-21.

[9] Fischer-Servi, G., "On modal logics with an intuitionistic base", Studia Logica 36 (1977): 141-149. DOI: 10.1007/BF02121259

[10] Fischer-Servi, G., "Semantics for a class of intuitionistic modal calculi", pages 59-72 in Italian Studies in the Philosophy of Science, M. L. Dalla Chiara (ed.), Reidel, Dordrecht, 1980.

[11] Fischer-Servi, G., "Axiomatizations for some intuitionistic modal logics", Rend. Sem. Mat. Univers. Polit., 42 (1984): 179-194.

[12] Font, J., "Modaility and possibility in some intuitionistic modal logics", Notre Dame Journal of Formal Logic 27, 4 (1986): 533-546.

[13] Gabbay, D. M., and L.L. Maksimova, Interpolation and Definability: Modal and Intuitionistic Logics, Claeendon press, Oxford, 2005.

[14] Maksimova, L. L., "Craig's theorem in superintuitionistic logics and amalgamated varieties of pseudoboolean algebras", Algebra and Logic, 16, 6 (1977): 427-455.

[15] Mihajlova, M., 'Reduction of modalities in several intuitionistic modal logics', Comptes rendus de l'Académie Bulgaire des Sciences 33 (1980): $743-745$.

[16] Segerberg, K., "Propositional logics related to Heyting's and Johansson's", Theoria, 34, 1 (1968): 26-61. DOI: 10.1111/j.1755-2567.1968.tb00337.x

[17] Sotirov, V., "Modal theories with intuitionistic logic", pages 139-171 in Mathematical Logic, Proc. of the Conference Dedicated to the memory of A. A. Markov (1903-1979), Sofia, September 22-23, Bulgarian Acad. of Sc., 1984. 
[18] Vakarelov, D., "Consistency, completeness and negation", pages 328-363 in Paraconsistent Logics: Essays on the Inconsistent, G. Priest, R. Routley, and J. Norman (eds.), Filosophia, 1989.

[19] Wolter, F., and M. Zakharaschev, "On the relation between intuitionistic and classical modal logics", Algebra and Logic, 36, 2 (1997): 121-155. DOI: $10.1007 / \mathrm{BF} 02672476$

[20] Wolter, F., and M. Zakharaschev, "Intuitionistic modal logics", pages 227-238 in Logical Foundations of Mathematics, A. Cantini, E. Casari, and P. Minari (eds.), Synthese Library, Kluwer, 1999.

[21] Yankov, V. A., "The calculus of weak law of excluded middle" (in Russian), Izv. Ak. Nauk SSSR Ser. Mat., 32, 5 (1968): 1044-1051.

SERGEy Drobyshevich

Sobolev Institute of Mathematics and Novosibirsk State University

Novosibirsk, 630090, Russia

drobs@math.nsc.ru 